\title{
The effect of the elliptical ratio on the tubular energy absorber subjected to lateral loading under quasistatic conditions
}

\author{
Ahmad Baroutaji ${ }^{1 a}$ \& Abdul Ghani Olabi \\ 1 School of mechanical and manufacturing engineering, Dublin city university, Dublin, Ireland
}

\begin{abstract}
:
Tubular systems are proposed to be used as energy absorber because they are cheap and easy to manufacture; recently some researchers use the elliptical tube as energy absorber.

In this work, the influence of elliptical ratio ( $r=D 1 / D 2)$ on energy absorption capability and load carrying capacity and stress of mild steel elliptical tubes has been investigated both experimentally and numerically, the experimental analyses conducted by using Zwick Type BT1-FB050TN testing instrument.

This machine is universal instrument for performing tensile test and compression test, Fig (1) and bending test and it is consider as an important machine for measuring the mechanical properties of materials and structures. The loading frame consist of two vertical lead screws, a moving crosshead and an upper and lower bearing plate which bears the load of the lead screws. The maximum capacity of the loading frame attached to the table mounted unit is $50 \mathrm{KN}$ In this study a velocity between 3$10 \mathrm{~mm} / \mathrm{min}$ was applied to the moving component to ensure the quasistatic conditions whereas velocities between $0.5 \mathrm{~mm} / \mathrm{min}$ and $15 \mathrm{~mm} / \mathrm{min}$ have been used by many researchers to simulate the quasi-static lateral compression of tubes between various indenters [1-2]. In addition to the experimental work, computational method using ANSYS is used to predict the loading and response of such tubes where series of models was performed with elliptical ratios ranging from 0.5 to 1.5 . Comparison of numerical and experimental forcedeflection response is presented.
\end{abstract}

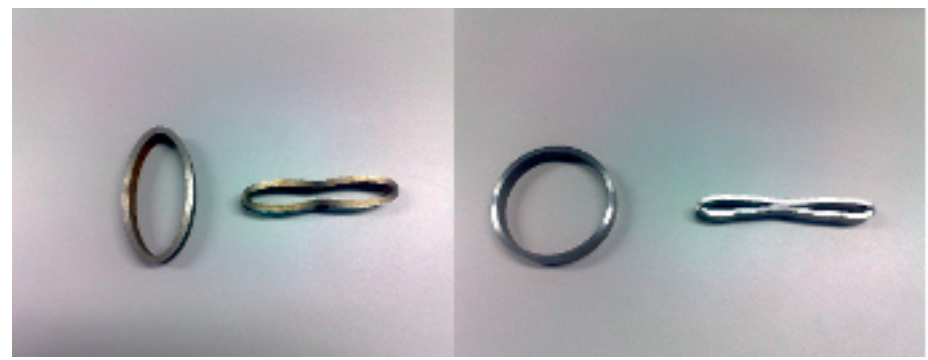

Fig (1): elliptical and circular tube before and after the compression test.

a e-mail : ahmad.baroutaji2@mail.dcu.ie

This is an Open Access article distributed under the terms of the Creative Commons Attribution-Noncommercial License 3.0, which permits unrestricted use, distribution, and reproduction in any noncommercial medium, provided the original work is properly cited. 
It has been found that with changing the elliptical ratio of the tube the loaddeflection curve change and this leads to change the energy absorbed by tube, the changing of the geometrical shape of the tube leads to change the volume of this tube and hence the mass. By reducing the elliptical ratio to 0.5 the tube will absorb $43.3 \%$ more energy and the system will gain $102 \%$ more in terms of specific energy, fig (2).

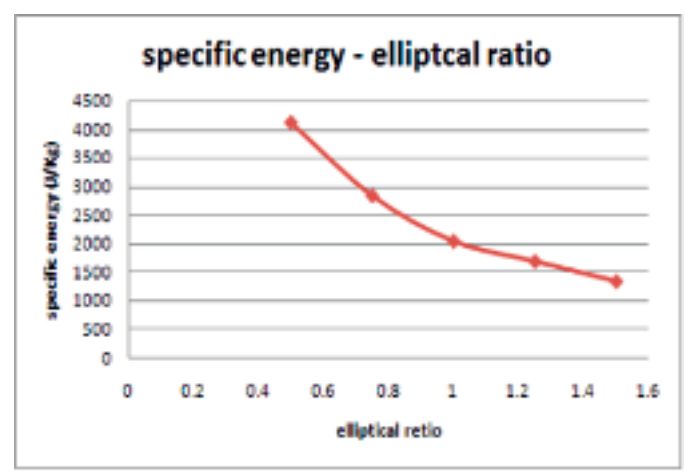

Fig (2): relationship between specific energy and elliptical ratio.

\section{Keywords:}

Energy Absorber, ANSYS, Quasi-static analyses, compression test, elliptical tube

\section{References}

[1] Reddy, Y.R., S. (1979), "Lateral compression of tubes and tube-systems with side constraints", Int.J.Mech.Sci., Vol.21 (3), pp. 187-199.

[2] Morris, E., Olabi, A.and Hashmi, M., (2007), "Lateral crushing of circular and non-circular tube systems under quasi-static conditions", Journal of Materials Processing Tech., Vol.191 (1-3), pp.

132-135. 